\title{
The effect of hepatic or renal impairment on the pharmacokinetics of edivoxetine, a selective norepinephrine transporter reuptake inhibitor
}

\author{
William Kielbasa $\cdot$ Eshetu Tesfaye $\cdot$ Debra Luffer-Atlas • \\ Malcolm I. Mitchell • Michael A. Turik \\ Received: 7 June 2013 / Accepted: 28 July 2013 / Published online: 17 August 2013 \\ (C) The Author(s) 2013. This article is published with open access at Springerlink.com
}

\begin{abstract}
Purpose To assess the impact of hepatic or renal impairment on the pharmacokinetics (PK) of edivoxetine.

Methods Two separate multi-center, open-label studies with males and females were conducted. Subjects were categorized according to their hepatic function, determined by the ChildPugh classification, or renal function, determined by creatinine clearance using the Cockcroft-Gault equation. Subjects received a single dose of $18 \mathrm{mg}$ in the hepatic impairment study or $6 \mathrm{mg}$ in the renal impairment study. Noncompartmental PK parameters were computed from the edivoxetine plasma concentration-time data.

Results In the hepatic study, the geometric least squares mean (GLSM) and $90 \%$ confidence interval (CI) of the ratio [impaired : normal] of area under the concentration versus time curve from time zero to infinity $(\mathrm{AUC} 0-\infty$; $\mathrm{h} \times \mathrm{ng} / \mathrm{mL})$ was $1.24(0.93,1.64)$ in the mild, $1.60(1.21,2.12)$ in the moderate, and $1.70(1.28,2.24)$ in the severe group. In the renal impairment study, the GLSM $(90 \% \mathrm{CI})$ of the ratio [impaired : normal] of AUC $0-\infty$ was $1.13(0.73,1.73)$ in mild, 1.90 $(1.28,2.82)$ in moderate, $1.55(0.94,2.55)$ in severe, and $1.03(0.66,1.59)$ in ESRD groups. Overall, the GLSM of the
\end{abstract}

The clinical trial registry number for the study conducted in subjects with compromised hepatic function is NCT01241435. Note that the Phase I trial conducted in subjects with compromised renal function is not included in a public registry.

W. Kielbasa $(\bowtie) \cdot$ D. Luffer-Atlas $\cdot$ M. A. Turik

Lilly Corporate Center, Eli Lilly and Company, Indianapolis, IN 46285, USA

e-mail: wkielbasa@lilly.com

E. Tesfaye

Lexicon Pharmaceutical, Inc., 8800 Technology Forest Place,

The Woodlands, TX 77381, USA

M. I. Mitchell

Erl Wood Manor, Eli Lilly and Company UK, Windlesham,

Surrey GU20 6PH, UK ratio [impaired : normal] of $\mathrm{C}_{\max }$ was slightly less than or approximately 1 across the hepatic and renal impairment groups. Across both studies, there were no clinically significant changes in vital signs and laboratory values, the adverse events were mild in severity and mostly related to nervous system and gastrointestinal disorder-related events.

Conclusions PK changes in subjects with hepatic or renal impairment were of small magnitude and did not appear to impact overall subject tolerability. Daily dosing of edivoxetine in a larger population of impaired subjects, including those with dual impairment, would aid in establishing edivoxetine tolerability and PK in a clinical practice scenario.

Keywords Edivoxetine $\cdot$ Pharmacokinetics .

Hepatic impairment · Renal impairment

\section{Introduction}

Edivoxetine (LY2216684 HCl; 2-Morpholinemethanol, $\alpha$-[(5-fluoro-2-methoxyphenyl) methyl]- $\alpha$-[tetrahydro-2Hpyran-4-yl]-, hydrochloride, [ $\alpha \mathrm{R}, 2 \mathrm{~S}]$ ) is a potent and highly selective norepinephrine reuptake inhibitor being evaluated as adjunctive treatment for adult patients with major depressive disorder (MDD) who are taking a selective serotonin reuptake inhibitor. Edivoxetine was studied in two double-blind investigations in adult patients with MDD [1,2] and an open-label study in pediatric patients with attention deficient hyperactivity disorder [3, 4].

The patient population for edivoxetine potentially includes those with hepatic or renal impairment, which might affect the pharmacokinetics (PK) of edivoxetine and may lead to changes in the safety, tolerability, and/or efficacy of the drug. Two distinct studies were conducted to assess the impact of hepatic impairment and renal impairment on the PK of edivoxetine. In the hepatic impairment study, the primary objective was to evaluate the PK of edivoxetine in subjects 
with mild, moderate, or severe hepatic impairment compared to subjects with normal hepatic function after a single oral dose of $18 \mathrm{mg}$ edivoxetine. In the renal impairment study, the primary objective was to evaluate the PK of edivoxetine in subjects with mild, moderate, and severe renal impairment or end-stage renal disease (ESRD) compared to subjects with normal renal function after a single oral dose of $6 \mathrm{mg}$ edivoxetine. A secondary objective of the renal impairment study was to assess the impact of dialysis on the PK of edivoxetine in subjects with ESRD. Both of these studies assessed the safety and tolerability of edivoxetine. The results of these investigations are reported herein.

\section{Methods}

Clinical study designs

\section{Hepatic impairment}

This study was an open-label, single-dose, parallel-design, multi-center study involving subjects who were categorized into four groups by their hepatic function, as defined by the Child-Pugh criteria [5, 6]: normal hepatic function, hepatic impairment due to liver cirrhosis classified as mild (ChildPugh A), hepatic impairment due to liver cirrhosis classified as moderate (Child-Pugh B), and hepatic impairment due to liver cirrhosis classified as severe (Child-Pugh C). At the time of screening, subjects were between 40 and 64 years old with a body mass index (BMI) of 17.4 to $35 \mathrm{~kg} / \mathrm{m}^{2}$. Subjects with normal hepatic function were required to be healthy as determined by medical history and a physical examination and to have clinical laboratory test results within normal reference ranges or with acceptable deviations deemed to be without clinical significance. Efforts were made to match subjects across groups in terms of age ( \pm 10 years), weight $( \pm 10 \mathrm{~kg})$, and sex. Each subject was screened up to 30 days prior to enrollment. Subjects were admitted to the Clinical Research Unit (CRU) on the day prior to dosing of edivoxetine and resided at the CRU for at least $72 \mathrm{~h}$ after edivoxetine was administered, except for severe hepatic impairment subjects who remained at the CRU for at least $120 \mathrm{~h}$ after edivoxetine was administered. Administration of the doses was preceded by a light breakfast given within $1 \mathrm{~h}$ of dosing. A total dose of $18 \mathrm{mg}$ of edivoxetine was given as three 6-mg tablets. Approximately 7 days following discharge from the study each subject returned for a follow-up visit.

\section{Renal impairment}

This study was a multi-center, open-label, 1-period study involving subjects who were categorized by their renal function as normal, and mild, moderate, or severe renal impairment, or as ESRD undergoing hemodialysis, based on estimated creatinine clearance $\left(\mathrm{CL}_{\mathrm{CR}}\right)$ by the Cockcroft-Gault (CG) equation as described in the 2010 FDA guidance [7]. Normal, mild, moderate, and severe renal function was defined by an estimated $\mathrm{CL}_{\mathrm{CR}}$ of $\geq 90 \mathrm{~mL} / \mathrm{min}, 60$ to $89 \mathrm{~mL} / \mathrm{min}$, 30 to $59 \mathrm{~mL} / \mathrm{min}$, and 15 to $29 \mathrm{~mL} / \mathrm{min}$, respectively. Subjects were 37 to 78 years of age with a BMI of 20.2 to $38.7 \mathrm{~kg} / \mathrm{m}^{2}$ at the time of screening. Subjects with normal renal function were required to be healthy as determined by medical history and a physical examination, with clinical laboratory test results within normal reference ranges or with acceptable deviations deemed to be without clinical significance. Efforts were made to match subjects across groups in terms of age ( \pm 10 years), weight $( \pm 10 \mathrm{~kg})$, sex, and race. Each subject was screened up to 21 days prior to enrollment. Renal impairment was assessed using the serum creatinine level when the subjects were admitted to the CRU. The ESRD subjects on hemodialysis were required to have been receiving hemodialysis for at least 3 months prior to enrollment.

Subjects were admitted to the CRU the night before they were scheduled to receive edivoxetine. After an 8-hour fast from food and a 1-hour water restriction, subjects not on hemodialysis were given a single 6-mg tablet of edivoxetine followed by a 4-hour fast. Water was restricted for $2 \mathrm{~h}$ postdose as well. Those subjects on hemodialysis received $6 \mathrm{mg}$ edivoxetine followed by a 4-hour fast on two separate occasions to allow for a comparison of the effect of hemodialysis on edivoxetine PK. Subjects completed a dialysis session, and then waited at least $18 \mathrm{~h}$ before taking the first 6-mg dose of edivoxetine. Approximately $48 \mathrm{~h}$ following edivoxetine administration subjects resumed their dialysis cycle. After at least 1 week from edivoxetine administration, the second 6-mg dose of edivoxetine was administered approximately $2 \mathrm{~h}$ prior to the start of a dialysis session. To eliminate potential variation arising from different types of hemodialysis membranes, only high-flux polysulfone membranes were used for hemodialysis. All subjects were discharged from the CRU after 24-hour postdose procedures at the discretion of the investigator. A follow-up visit occurred approximately 7 days following discharge for all subjects.

\section{Pharmacokinetics}

\section{Blood sampling}

In the hepatic study, blood samples were collected for the measurement of plasma concentrations of edivoxetine up to $72 \mathrm{~h}$ postdose for all subjects, except those with severe hepatic impairment whose samples were collected up to $120 \mathrm{~h}$ postdose. In the renal study, blood samples were collected for 
the measurement of the plasma concentration of edivoxetine up to $72 \mathrm{~h}$ postdose for all subjects.

\section{Bioanalysis}

Plasma samples were analyzed for edivoxetine concentrations using a validated liquid chromatography assay with tandem mass spectrometry detection. Plasma samples were analyzed at Covance Laboratories Inc. located in Madison, Wisconsin, USA. The lower limit of quantification was $0.20 \mathrm{ng} / \mathrm{mL}$, and the upper limit of quantification was $100.00 \mathrm{ng} / \mathrm{mL}$. The interassay accuracy during validation ranged from $-3.5 \%$ to $2.7 \%$. The interassay precision described as percent relative standard deviation during validation ranged from $1.4 \%$ to $4.5 \%$. Edivoxetine was stable in plasma for up to 364 days when stored at approximately $-20{ }^{\circ} \mathrm{C}$ and $-70{ }^{\circ} \mathrm{C}$.

\section{Pharmacokinetic and statistical analysis}

Pharmacokinetic parameters were computed by standard noncompartmental methods of analysis using WinNonlin Professional Version 5.2. The PK parameters computed included area under the concentration versus time curve from time zero to infinity (AUC0- $\infty$; unit: $\mathrm{h} \times \mathrm{ng} / \mathrm{mL}$ ), half-life associated with the terminal rate constant $\left(\mathrm{t}_{1 / 2} ;\right.$ unit: $\left.\mathrm{h}\right)$, apparent total body clearance $(\mathrm{CL} / \mathrm{F}$; unit: $\mathrm{L} / \mathrm{h})$, and apparent volume of distribution during the elimination phase $\left(\mathrm{V}_{\mathrm{Z}} / \mathrm{F}\right.$; unit: $\left.\mathrm{L}\right)$. The maximum observed drug concentration $\left(\mathrm{C}_{\max } ;\right.$ unit: $\left.\mathrm{ng} / \mathrm{mL}\right)$ and the time of $\mathrm{C}_{\max }\left(\mathrm{t}_{\max }\right.$; unit: $\mathrm{h}$ ) was determined from the PK profile.

Statistical analysis was conducted on $\log$ transformed AUC0- $\infty$ and $\mathrm{C}_{\max }$ using an analysis of variance model with group as a fixed factor. The primary outcome of the analysis was the ratio of geometric least squares means (GLSM) and their corresponding $90 \%$ confidence intervals (CI) of AUC0- $\infty$ and $\mathrm{C}_{\max }$ between the renal impairment or hepatic impairment groups versus the respective normal function group.

A continuous analysis using linear regression was performed to evaluate the relationship between estimated $\mathrm{CL}_{\mathrm{CR}}$ and edivoxetine $\mathrm{CL} / \mathrm{F}$ from the renal study. Similarly, Child-Pugh score and edivoxetine CL/F from the hepatic study was evaluated.

\section{Safety and tolerability}

For both studies, safety and tolerability were assessed by means of vital signs measurement, physical examinations, clinical laboratory tests, electrocardiogram, and adverse event (AE) recording.

\section{Results}

Demographics

The demographic data for subjects participating in the hepatic impairment study $(N=36)$ and renal impairment study $(N=42)$ is shown in Table 1.

Pharmacokinetics

\section{Hepatic impairment}

The PK profiles resulting from a single oral dose of $18 \mathrm{mg}$ of edivoxetine administered to subjects with normal hepatic function and varying degrees of hepatic impairment are illustrated in Fig. 1. An increase in AUC0- $\infty$ and $t_{1 / 2}$, a decrease in $\mathrm{CL} / \mathrm{F}$ and $\mathrm{C}_{\max }$, and minimal to no change in $\mathrm{t}_{\max }$ and $\mathrm{V}_{\mathrm{Z}} / \mathrm{F}$ was observed in subjects with hepatic impairment (Table 2). The GLSM $(90 \% \mathrm{CI})$ of the ratio [impaired : normal] of AUC0- $\infty$ was $1.24(0.93,1.64)$ in the mild, $1.60(1.21,2.12)$ in the moderate, and $1.70(1.28,2.24)$ in the severe group. The GLSM $(90 \% \mathrm{CI})$ of the ratio [impaired : normal] of $\mathrm{C}_{\max }$ was $0.85(0.69,1.03)$ in the mild, $0.89(0.73,1.09)$ in the moderate, and $0.74(0.61,0.91)$ in the severe group.

The relationship between Child-Pugh score and edivoxetine $\mathrm{CL} / \mathrm{F}$ is illustrated in Fig. 2. The estimate of the slope of the linear regression was -1.80 with a $p$-value of 0.0781 and a correlation coefficient of 0.3665 . These results indicate the slope of the linear regression was not statistically significant, and there was a lack of an apparent correlation between Child-Pugh score and CL/F.

\section{Renal impairment}

The PK profiles resulting from a single oral dose of $6 \mathrm{mg}$ of edivoxetine administered to subjects with normal renal function and varying degrees of renal impairment are illustrated in Fig. 3. In general, an increase in AUC0- $\infty$ and $t_{1 / 2}$, a decrease in $\mathrm{CL} / \mathrm{F}$, and minimal to no change in $\mathrm{t}_{\max }, \mathrm{C}_{\max }$ and $\mathrm{V} / \mathrm{F}$ was observed in subjects with renal impairment (Table 2). The GLSM $(90 \% \mathrm{CI})$ of the ratio [impaired : normal] of AUC0- $\infty$ was $1.13(0.73,1.73)$ in mild, $1.90(1.28,2.82)$ in moderate, $1.55(0.94,2.55)$ in severe, and $1.03(0.66,1.59)$ in ESRD groups. The AUC0- $\infty 90 \%$ CIs of all the renal impairment groups included unity except for the moderate group. The GLSM $(90 \% \mathrm{CI})$ of the ratio [impaired : normal] of $\mathrm{C}_{\max }$ was $0.79(0.54,1.16)$ in mild, $1.06(0.75,1.51)$ in moderate, $1.10(0.70,1.72)$ in severe, and $0.84(0.56,1.24)$ in ESRD. Hemodialysis did not appear to have any meaningful impact on the PK of edivoxetine in ESRD subjects (Table 2). For the two subjects with ESRD not receiving hemodialysis (see Table 1), the AUC0- $\infty$ was $133 \mathrm{~h} \times \mathrm{ng} / \mathrm{mL}$ and $409 \mathrm{~h} \times \mathrm{ng} / \mathrm{mL}$, 


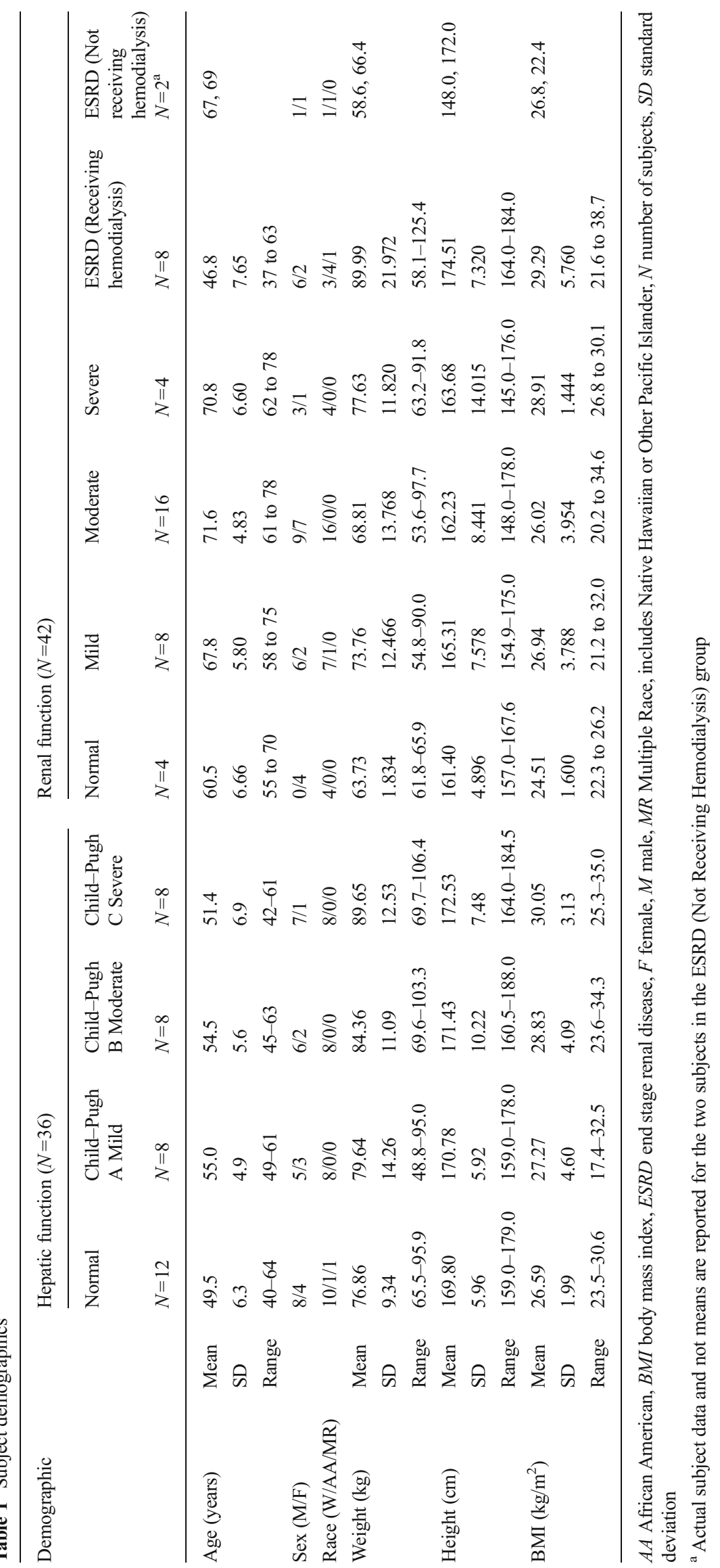



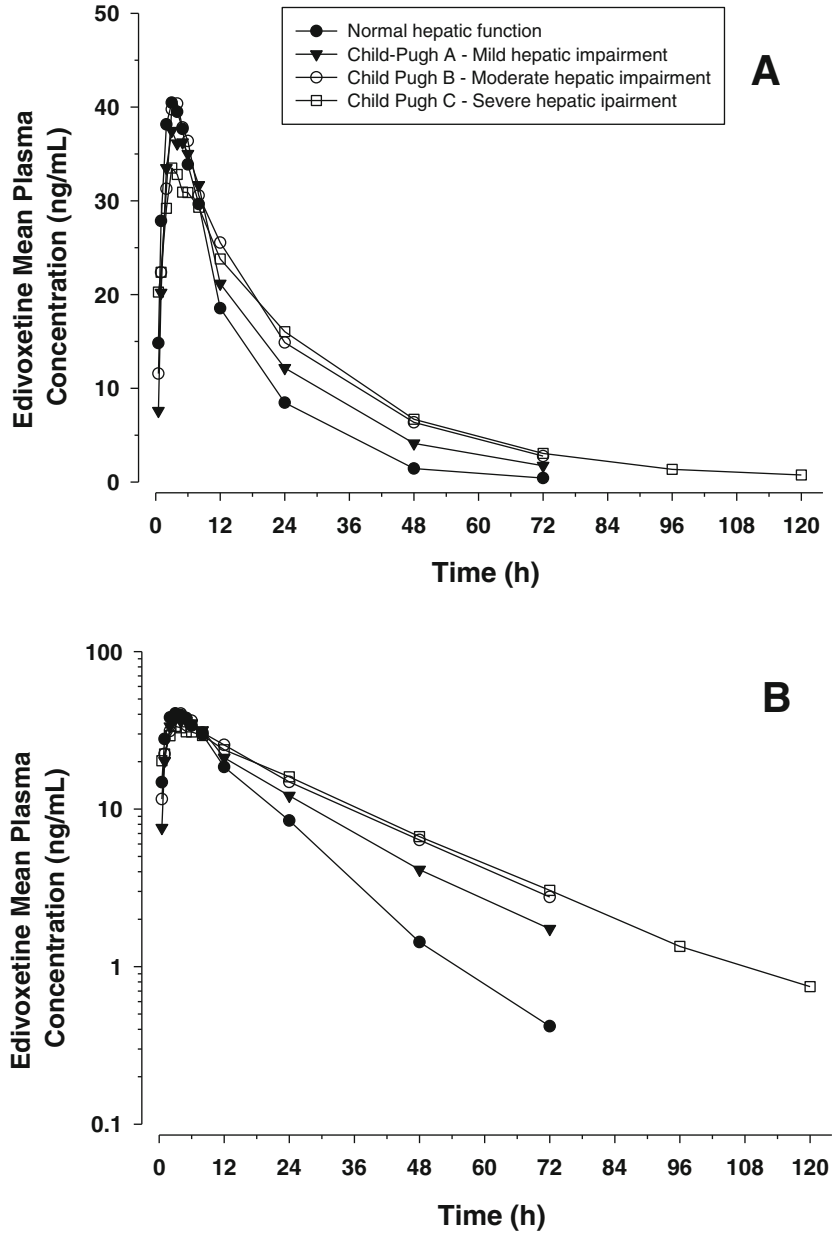

Fig. 1 Arithmetic mean edivoxetine plasma concentration-time profiles after a single oral administration of $18 \mathrm{mg}$ edivoxetine to subjects with varying degrees of hepatic function (upper panel a: linear scale; lower panel b: semi-logarithmic scale)

and the Cmax was $18.4 \mathrm{ng} / \mathrm{mL}$ and $18.6 \mathrm{ng} / \mathrm{mL}$. These two subjects were not included in the summary of PK parameters (Table 2) or in the statistical analysis of variance model, but were included in the continuous linear regression as shown in Fig. 4.

The relationship between $\mathrm{CL}_{\mathrm{CR}}$ and edivoxetine $\mathrm{CL} / \mathrm{F}$ is illustrated in Fig. 4. The slope of the linear regression was statistically different from zero (p-value: 0.0457 ) and the correlation coefficient was 0.3449 . Based on linear regression, the $y$-intercept $\left(\mathrm{CL}_{\mathrm{CR}}=0\right.$; no theoretical renal function) for $\mathrm{CL} / \mathrm{F}$ was $15.7 \mathrm{~L} / \mathrm{h}$, which is within range of $\mathrm{CL} / \mathrm{F}$ of many subjects in this study across varying renal impairment groups. The model predicted mean CL/F (95\% predicted CI) increased by a factor of approximately 1.8 , from $16.6 \mathrm{~L} / \mathrm{h}(0$, $36.9)$ to $30.3 \mathrm{~L} / \mathrm{h}(9.13,51.5)$, when the $\mathrm{CL}_{\mathrm{CR}}$ increased by a factor of approximately 20 indicating that a considerable change in $\mathrm{CL}_{\mathrm{CR}}$ is needed to have an impact on $\mathrm{CL} / \mathrm{F}$.

Urine was not collected and analyzed for concentrations of edivoxetine in the renal impairment study. Previous evaluations demonstrated that unmetabolized edivoxetine was not principally eliminated from the body via the urine $(<18 \%$ of the dose) and that edivoxetine renal clearance contributed about $20 \%$ of total body clearance. The degree to which the kidney plays a role in edivoxetine elimination is reflected by the effect of renal impairment on edivoxetine PK in this study.

Plasma protein binding evaluations were not conducted in the renal impairment study. It had been determined previously that the extent of edivoxetine binding to human plasma protein was approximately $65 \%$ and drug concentration independent. According to the FDA guidance [7], a potential alteration in protein binding due to impaired renal function for drugs with an extent of binding less than $80 \%$ would likely lead to a relatively minor change in the unbound fraction of circulating drug. As such, total edivoxetine plasma concentrations were deemed to be adequate and were exclusively used in the PK analyses in this study.

Safety and tolerability

\section{Hepatic impairment}

No deaths occurred, and no subjects discontinued because of an AE. Of the 36 subjects who received edivoxetine, 20 (56\%) experienced a total of 44 treatment-emergent AEs (TEAEs), with 16 (44\%) of these subjects reporting 31 AEs that were considered by the investigator to be related to edivoxetine. In each hepatic function group, the majority of AEs reported were mild in severity, with no serious or severe adverse events reported. Moderate AEs were reported by three subjects during the study, including vomiting reported by a subject in the normal group, somnolence reported by a subject with mild hepatic impairment, and tinnitus, feeling abnormal, headache, and fatigue reported by a subject with moderate hepatic impairment. Across all groups, the most commonly reported AEs were nervous system disorders including dizziness, headache, and somnolence, and gastrointestinal disorders including nausea, constipation, vomiting, abdominal discomfort, and dry mouth. No clinically significant changes in laboratory values and vital signs were reported.

\section{Renal impairment}

No deaths occurred, and no subjects discontinued because of an AE. There was one report of a serious AE by a subject with ESRD receiving hemodialysis that developed a clotted fistula, classified as a malfunctioning vascular device/graft, which required hospitalization to replace the device with a permanent catheter. This event was not judged by the investigator to be related to edivoxetine. Of the 42 subjects who received edivoxetine, 14 reported TEAEs. Of the 14 subjects that reported TEAEs, eight reported TEAEs that were judged by the investigator to be related to edivoxetine. Most TEAEs were of mild severity, and the most common TEAEs were 


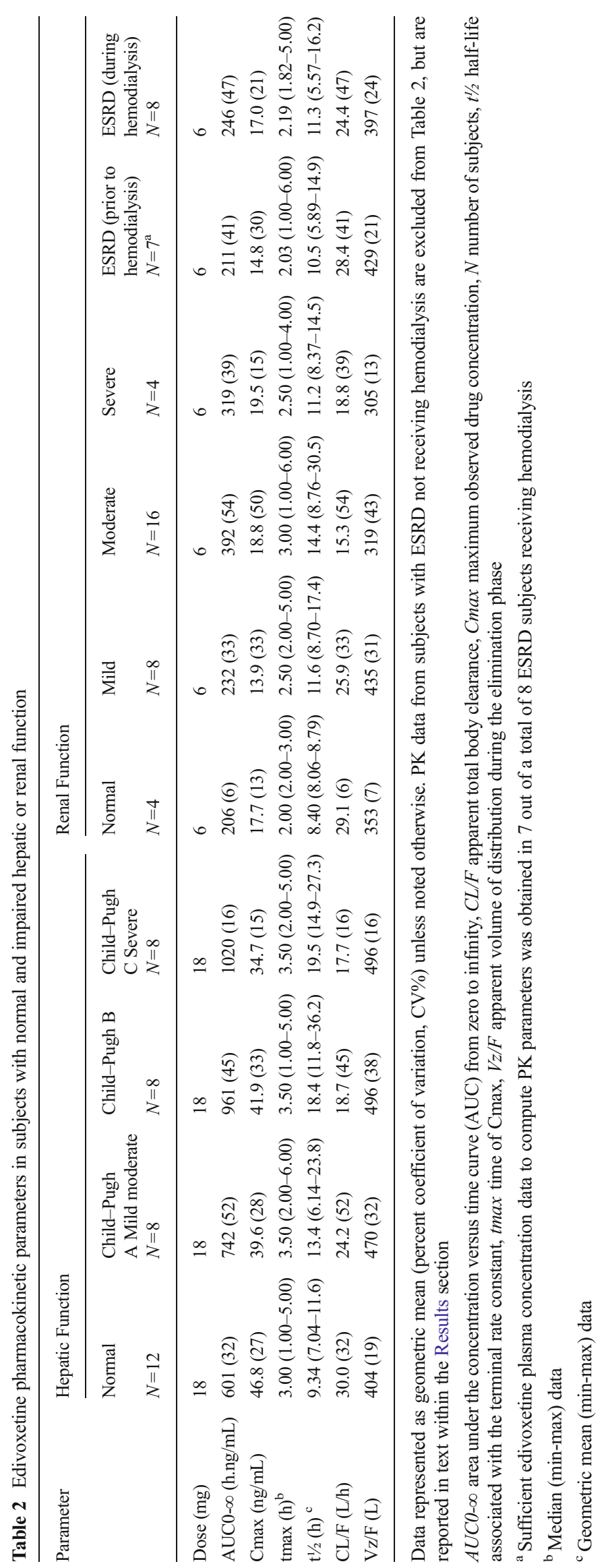




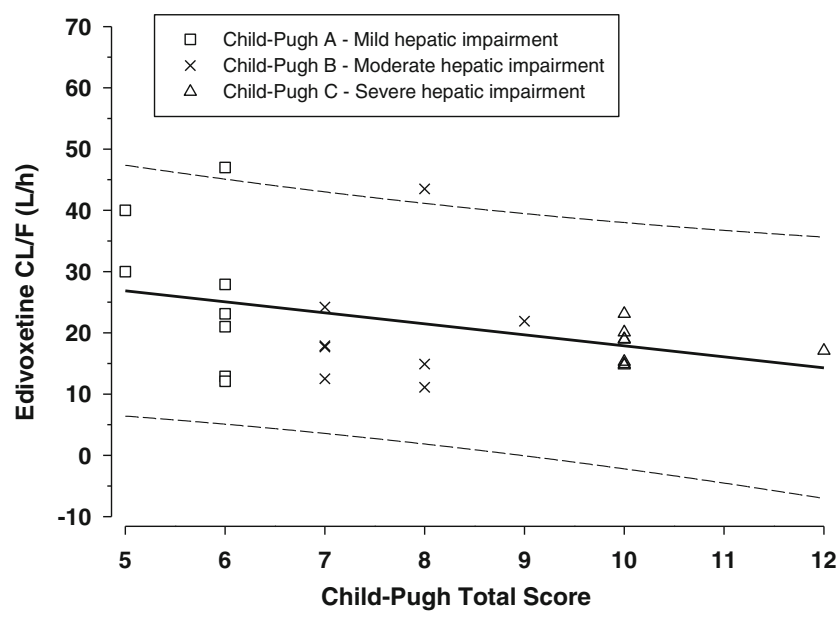

Fig. 2 Relationship between Child-Pugh total score and edivoxetine oral clearance $(\mathrm{CL} / \mathrm{F})$

nausea and dizziness. One subject with ESRD reported 2 TEAEs of urinary hesitation judged by the investigator to be moderate in severity. While there were no TEAEs reported in
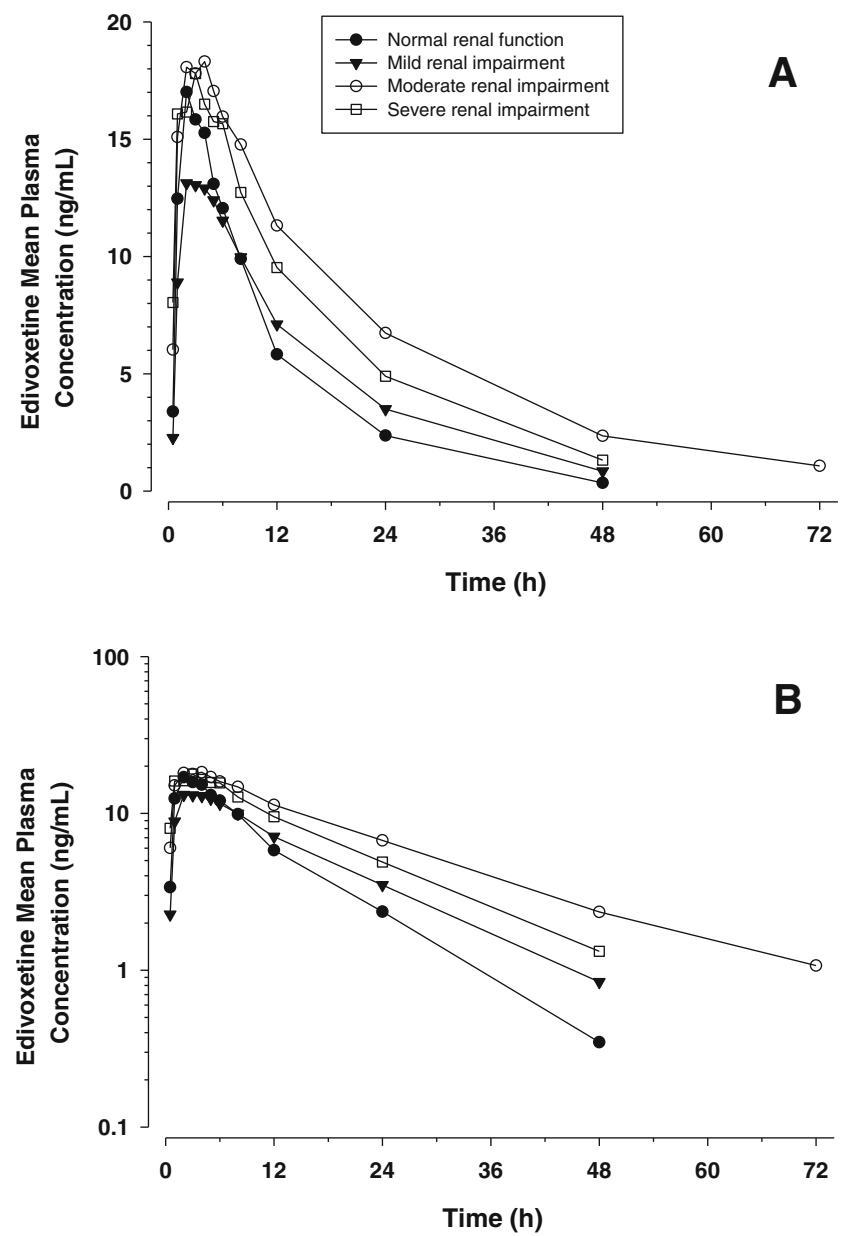

Fig. 3 Arithmetic mean edivoxetine plasma concentration-time profiles after a single oral administration of $6 \mathrm{mg}$ edivoxetine to subjects with varying degrees of renal function (upper panel a: linear scale; lower panel b: semi-logarithmic scale)

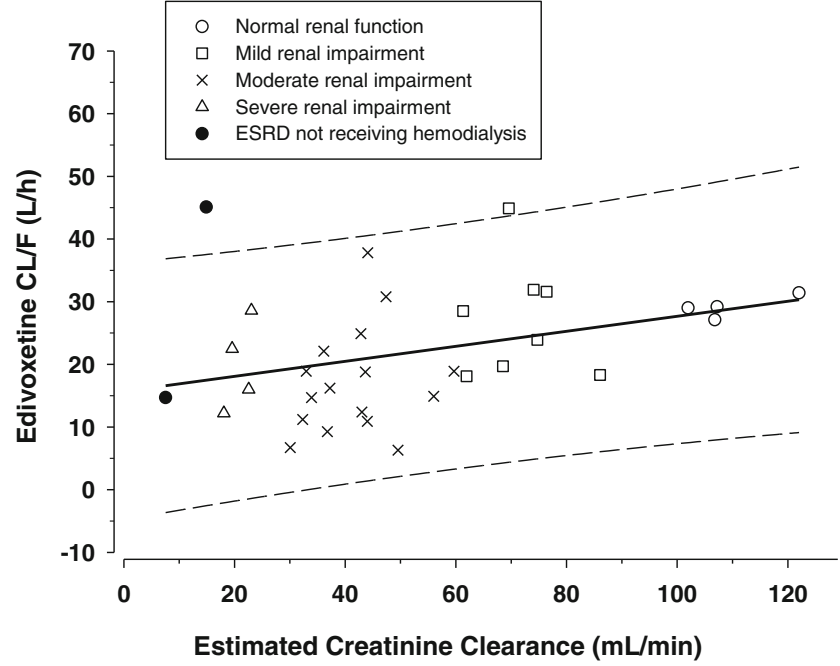

Fig. 4 Relationship between estimated creatinine clearance and edivoxetine oral clearance (CL/F)

the normal group, the number of reported AEs did not increase with the degree of renal impairment. No clinically significant changes in laboratory values or vital signs were reported. In subjects with renal impairment, predicted out-of-range laboratory and ECG parameters were noted, but did not appear to impact overall subject safety.

\section{Discussion}

The doses of edivoxetine evaluated as adjunctive treatment for adults with MDD range from $6 \mathrm{mg}$ to $18 \mathrm{mg}$ [8-11]. The doses investigated in the renal and hepatic studies were $6 \mathrm{mg}$ and $18 \mathrm{mg}$, respectively. The dose in the renal study was lower because understanding of the safety, PK, and pharmacodynamics of edivoxetine at the time the renal study was conducted was not as extensive relative to when the hepatic study was conducted; as a result, a conservative renal study design was implemented. Nevertheless, based on exposure and safety data obtained from subsequent clinical studies up to a top dose of $36 \mathrm{mg}$, the PK of edivoxetine appears to be concentration independent (linear clearance) and time independent (stationary). For these reasons, the results obtained from the renal and hepatic studies can be reasonably applied to a wide dose range of edivoxetine.

The heterogeneity of the demographics (Table 1) within the studies is worth noting. In the renal study, there was large variability in the number of subjects at each group, and the normal group was comprised of only female subjects, whereas the impaired groups contained mostly males that weighed more than the females. The age across groups was mostly similar, except for the ESRD group receiving hemodialysis, which was considerably younger than all other groups. In the hepatic study, the differences across groups based on age, race 
or sex were less prominent than in the renal study. Results of our analysis demonstrated that there is a slight trend for lower $\mathrm{CL} / \mathrm{F}$ when $\mathrm{CL}_{\mathrm{CR}}$ is reduced in renally impaired subjects or when the Child-Pugh score is increased in hepatically impaired subjects. Furthermore, a continuous regression analysis was used to evaluate the effect of renal or hepatic function on $\mathrm{CL} / \mathrm{F}$ due to the variability in demography across study groups that could possibly skew categorical results. Diversity in subject demographics was not anticipated to have a significant impact on PK based on a previous clinical investigation that showed edivoxetine exposure was only mildly increased in elderly subjects compared to young subjects, and within elderly subjects aged 65-81 years there was no difference in exposure [unpublished data]. Similarly, preliminary evaluations using a population PK analysis approach demonstrated that sex and body weight were not covariates of edivoxetine PK in adults [unpublished data]. For these reasons, the PK results obtained from the renal and hepatic impairment studies are considered to be valid.

In the hepatically impaired subjects, the increase in AUC0- $\infty$ was of small magnitude and was not appreciably different in the moderate and severe groups. A delay of 1 to 2 days to reach steady state (SS) plasma concentrations is expected in the moderate and severe groups due to the increase in $t_{1 / 2}$, but this change is not believed to have any clinical consequence on the safety or efficacy of the drug. An increase in bioavailability cannot be ruled out as a possible contributing factor to the increase in AUC0- $\infty$. A slight decrease was observed in $\mathrm{C}_{\max }$ and increase in $\mathrm{t}_{\max }$ in hepatically impaired subjects, which were consistent with a presumed decrease in the rate of edivoxetine absorption. While the exact mechanism for reduced edivoxetine $\mathrm{CL} / \mathrm{F}$ is unknown, it was previously established that edivoxetine is metabolized in the liver primarily by cytochrome P450 (CYP) 2D6 [12] and CYP3A enzymes and is excreted with metabolites into feces and, to a minor extent, in urine [unpublished data]. It is possible that the activity or the metabolic capacity of enzymes responsible for the metabolism of edivoxetine is compromised to some degree in hepatically impaired subjects. However, it should be noted that while the Child-Pugh scale is the most commonly used method for classifying subjects with hepatic impairment in clinical trials, this scale was not developed for the purpose of predicting drug elimination capacity. The analysis indicated that the correlation between Child-Pugh score and edivoxetine CL/F was not statistically significant and corroborates our categorical analysis of PK parameters across the impairment groups.

In renally impaired subjects, a modest increase in $\mathrm{t}_{1 / 2}$ and AUC $0-\infty$ was observed but was not correlated with the degree of renal impairment. Renal impairment can lead to alterations in bioavailability and nonrenal clearance by affecting pathways of intestinal and hepatic drug-metabolizing enzymes and transporters [13-16]. As CYP450 enzymes are known to metabolize edivoxetine, an increase in bioavailability and/or reduced elimination from the body (based on $\mathrm{t}_{1 / 2}$ ) may account for the observed increase in AUC $0-\infty$ in renally impaired subjects.

Following a single dose of edivoxetine, hepatic or renal impairment did not appear to impact overall subject tolerability in these studies. The AEs reported were similar across the renal and hepatic studies, mild in severity, and similar to previously reported clinical investigations with edivoxetine in healthy subjects that achieved similar plasma exposures. Although the data obtained did not raise any safety and tolerability concerns, the studies were conducted in a small patient population, of which subjects had either renal or hepatic impairment, but not both. Daily dosing of edivoxetine in a larger population of impaired subjects, including those with varying degrees of dual impairment, would aid in establishing edivoxetine tolerability and PK in a clinical practice scenario.

Acknowledgments The authors thank the study investigators at Elite Research Institute, Pivotal Research Centers, Orlando Clinical Research Center and Clinical Pharmacology of Miami, Inc., and Covance Laboratories Inc. for their participation in clinical trial activities. The authors also thank Tonya Quinlan for her support in preparing the manuscript. Editorial support was provided by Amy Burdan of INC Research ${ }^{\circledR}$ and was funded by Eli Lilly and Company.

Contribution of authors All authors provided substantial contributions to the conception and design of the clinical trials and were responsible for analysis and interpretation of data contained within this manuscript. William Kielbasa prepared the initial draft of the manuscript. All authors revised the manuscript critically for important intellectual content, and read and approved the final manuscript.

Conflict of interest WK, DLA, MIM, and MAT are employees and stockholders of Eli Lilly and Company. ET was an employee and stockholder of Eli Lilly and Company at the time this work was conducted.

Open Access This article is distributed under the terms of the Creative Commons Attribution License which permits any use, distribution, and reproduction in any medium, provided the original author(s) and the source are credited.

\section{References}

1. Dubé S, Dellva MA, Jones M, Kielbasa W, Padich R, Saha A, Rao P (2010) A study of the effects of LY2216684, a selective norepinephrine reuptake inhibitor, in the treatment of major depression. J Psychiatr Res 44(6):356-363. doi:10.1016/j.jpsychires. 2009.09.013

2. Pangallo B, Dellva MA, D’Souza DN, Essink B, Russell J, Goldberger C (2011) A randomized, double-blind study comparing LY2216684 and placebo in the treatment of major depressive disorder. J Psychiatr Res 45(6):748-755. doi:10.1016/j.jpsychires. 2011.03.014

3. Kielbasa W, Quinlan T, Jin L, Xu W, Lachno DR, Dean RA, Allen AJ (2012) Pharmacokinetics and pharmacodynamics of edivoxetine (LY2216684), a norepinephrine reuptake inhibitor, in pediatric 
patients with attention-deficit/hyperactivity disorder. J Child Adolesc Psychopharmacol 22(4):269-276. doi:10.1089/cap.2011.0151

4. Jin L, Xu W, Krefetz D, Gruener D, Kielbasa W, TauscherWisniewski S, Allen AJ (2013) Clinical outcomes from an open-label study of edivoxetine use in pediatric patients with attention-deficit/ hyperactivity disorder. J Child Adolesc Psychopharmacol 23(3):200 207. doi:10.1089/cap.2012.0016

5. Child CG, Turcotte JG (1964) Surgery and portal hypertension. Major Probl Clin Surg 1:1-85

6. Pugh RNH, Murray-Lyon IM, Dawson JL, Pietroni MC, Williams R (1973) Transection of the oesophagus for bleeding oesophageal varices. Br J Surg 60(8):646-649

7. Food and Drug Administration (2010) Draft guidance for industry pharmacokinetics in patients with impaired renal function - study design, data analysis, and impact on dosing and labeling. http:// www.fda.gov/downloads/Drugs/.../Guidances/UCM204959.pdf. Accessed 29 April 2013

8. Eli Lilly and Company. A safety study in patients with major depressive disorder. In: ClinicalTrials.gov [Internet]. Bethesda (MD): National Library of Medicine (US). 2000- [cited 2013 May 21]. Available from: http://clinicaltrials.gov/ct2/show/NCT01155661?term= LY2216684\&rank=21 NLM Identifier: NCT01155661

9. Eli Lilly and Company. A fixed dose study of adjunctive treatment to antidepressant therapy for adults with major depressive disorder. In: ClinicalTrials.gov [Internet]. Bethesda (MD): National Library of Medicine (US). 2000- [cited 2013 May 21]. Available from: http:// clinicaltrials.gov/ct2/show/NCT01173601?term=LY2216684\&rank= 24 NLM Identifier: NCT01173601
10. Eli Lilly and Company. A study in patients with major depressive disorder who are partial responders to selective serotonin reuptake inhibitor. In: ClinicalTrials.gov [Internet]. Bethesda (MD): National Library of Medicine (US). 2000- [cited 2013 May 21]. Available from: http://clinicaltrials.gov/ct2/show/NCT01185340?term= LY2216684\&rank=26 NLM Identifier: NCT01185340

11. Eli Lilly and Company. A study of LY2216684 in patients with major depression disorder. In: ClinicalTrials.gov [Internet]. Bethesda (MD): National Library of Medicine (US). 2000- [cited 2013 May 21]. Available from: http://clinicaltrials.gov/ct2/show/NCT01370499? term $=$ LY2216684\&rank=27 NLM Identifier: NCT01370499

12. Kielbasa W, Luffer-Atlas D, Wondmagegnehu E, Mitchell M, Turik M (2011) Investigation of the potential for PK interactions between edivoxetine, a norepinephrine reuptake inhibitor, and selective serotonin reuptake inhibitor antidepressants in humans. ASCPT abstract P11165 Clin Pharmacol Ther (suppl):S88

13. Dreisbach AW (2009) The influence of chronic renal failure on drug metabolism and transport. Clin Pharmacol Ther 86(5):553-556. doi:10.1038/clpt.2009.163

14. Nolin TD, Naud J, Leblond FA, Pichette V (2008) Emerging evidence of the impact of kidney disease on drug metabolism and transport. Clin Pharmacol Ther 83(6):898-903. doi:10.1038/clpt.2008.59

15. Sun H, Frassetto L, Benet LZ (2006) Effects of renal failure on drug transport and metabolism. Pharmacol Ther 109(1-2):1-11

16. Zhang Y, Zhang L, Abraham S et al (2009) Assessment of the impact of renal impairment on systemic exposure of new molecular entities: evaluation of recent new drug applications. Clin Pharmacol Ther 85(3):305-311. doi:10.1038/clpt.2008.208 